\title{
Ciphertext-Policy Attribute-based Keyword Search with Revocation in Cloud through LSSS Approach
}

\author{
Chih-Hung Wang ${ }^{1}$, Yu-Hsuan Chang ${ }^{2}$ \\ ${ }^{1,2}$ Department of Computer Science and Information Engineering, National Chiayi University
}

\begin{abstract}
In the cloud computing, attribute-based encryption and public key encryption with keyword search are two popular issues. Ciphertext-policy attribute-based encryption is famous for its fine-grained access control. Keyword Search over encrypted files is an important function in the actual situation. We particularly concern the problem of revocation. Additionally, several solutions of the dynamic attribute revocation have been proposed in previous papers. This paper first combines the revocation and keyword search issues. In our proposed scheme, Linear Secret-Sharing Scheme (LSSS) is used for the access policy with efficiency manner as well as to solve the keyword attribute revocation problem.
\end{abstract}

Keywords: Cloud computing, Attribute-based keyword search, Ciphertext policy, Revocation, Re-encryption

\section{Introduction}

Nowadays, people are used to store the data on the cloud. The advantage of using cloud storage lies in convenience. Through the cloud, you can use the data anywhere and you can easily share them with others.

More and more people concern the security of cloud server. If you upload your files to the cloud server, you cannot confirm that the file is only given to the person who you expects. So you must upload the encrypted file and ensure that only the valid data receiver can decrypt the ciphertext; it shows that even the cloud server has no authority to access user's file. If the cloud has been compromised and the stored files are leaked, the attacker only can get the encrypted files and cannot decrypt them due to the lack of valid keys.

For the good fine-grained access control, the attribute-based encryption [9] can be an appropriate implementation. In the ciphertext-policy [3] approach, the file is encrypted under attributes, and the user who has the attributes satisfying the access policy can decrypt the ciphertext.

Our Contribution: We proposed a CPABE-based keyword search mechanism with attribute revocation under Linear Secret-Sharing Scheme (LSSS) [12]. When the data owner needs to revoke the attribute of ciphertext, cloud service provider (CSP) can perform the re-encrypt algorithm on the keywords of the ciphertexts.

\section{Related Work}

\subsection{Attribute-based Encryption}

In 2005, Sahai and Waters [9] proposed the concept of attribute-based encryption (ABE). ABE is a kind of encryption system that uses the attributes as the keys of creating the ciphertext. Compared with other encryption methods, ABE has a nice property of fine-grained access control. Access structure can be established according to "and", "or" or threshold gate. ABE is related to attributes instead of the number and the identities of the users. The former increases the cost of encryption calculation and the latter may leak the privacy of the user.

$\mathrm{ABE}$ can be divided into two types: KPABE (Key Policy ABE) and CPABE (Ciphertext Policy ABE). In $\mathrm{KPABE}$, the file is encrypted by a set of attributes and the user's key is associated with the access structure. If the file's attributes satisfy the access structure of the user, he can decrypt the ciphertext and get the original file. Otherwise, the decryption will be failed. 
In CPABE [3], the file is encrypted by the access structure and user's key is associated with a set of attributes. The user can decrypt the ciphertext only if user's attributes satisfy the access structure of the file. $\mathrm{CPABE}$ is more practical in the application of the real-world situation.

In 2011, Waters [12] proposed an efficient CPABE scheme. It is the first paper that uses the linear secret sharing scheme (LSSS) to construct the access structure. LSSS can be more efficient than the original secret sharing schemes constructed by the polynomial method.

\subsection{Public Key Encryption with Keyword Search}

Keyword search is an important part of a cloud storage system. Without the keyword search, you need to find what you want by flipping through all data that satisfy the access structure. The data in the cloud must be encrypted due to the cloud usually being regarded as untrusted party. Recently, many papers that discuss about keyword search under the encrypted data have been proposed $[4,5,6,13]$.

The technology of public key encryption with keyword search (PEKS) in [4] means that the user A wants to send the encrypted message to the user B and thus A encrypts the keyword by B's public key. In this case, only B can search this encrypted message by using the same keyword. In 2011, Li et al. proposed the authorized private keyword search (APKS) scheme [6]. In their scheme, there are multi-owners and multi-users. They used hierarchical predicate encryption to compare trap and cap, then they proposed APKS+ scheme which can further prevent the dictionary attack.

In [13], the authors proposed the first verifiable attribute-based keyword search (VABKS) scheme. In their scheme, it can search the data by keyword from cloud, and the user can verify the data from cloud to check whether it is complete or not. They put the concept of verifiable keyword search into both KPABE and CPABE. On the search part, the authors used three variables to make the equation for the trapdoor from data owner and the token from the user. The equation holds if the attributes are satisfied and the keywords in both trapdoor and token are matched. The secret of the user will not be leaked during the keyword query. The data owner generates the trapdoor while encrypting the data, and the user generates the keyword upon searching the data. The cloud service provider (CSP) will compare the trapdoor and token. If they are matched, CSP will return the corresponding ciphertext. On the part of verification, the signature and bloom filters were applied to achieve the goal.

In 2015, Dong et al. [5], based on VABKS scheme [13], split the constructions of Encrypt and TokenGen so that the user can run the TokenGen by online and offline. The user can calculate the part unrelated to the keyword in the offline phase, and then complete the keyword part when he wants to search.

\subsection{Revocation}

A possible situation, one day the data owner wants to change the access control of an encrypted file. At that moment, if the system did not consider the revocation procedure, the data owner needs to remove the original ciphertext and rebuild a new ciphertext by the modified access policy. On the other hand, if the system can properly handle the revocation process, the CSP can efficiently perform the re-encryption on the ciphertext. The data owner just needs to give the CSP the modified access structure for re-encryption. In 2011, Liang et al. [8] proposed the CPABE scheme with revocation. In their scheme, it is necessary to include all users in the setup algorithm because they use a list to check who will be revoked. To change the revocation list at slot time $t$ is what they want to do for the problem of revocation. The paper is based on mobile social network, so it uses the slot time. In the slot time $t$, the revocation list is active. After that time, the revocation list is invalid.

In [7], the authors proposed a proxy re-encryption scheme that the proxy is responsible for re-encrypting the file which the data owner wants to change the access structure. Their paper is based on Water [12] and can be secure against chosen-ciphertext attacks. Further, the authors used TCR hash function to sign the ciphertext, and used a verification key to check those signatures. 
In 2014, the scheme in [14] deal with the problem about the revocation for the attributes of the ciphertext. Their scheme allows the data owner to drop the attributes in the encrypted file by using the re-encryption procedure. And in their structure, it does not need to update the keys of all users who have revoked attributes. The server in the scheme has its own key that can be used to re-encrypt the ciphertext so that the system can achieve the attribute revocation and reduce the computation and communication costs for the authority and users.

In [10], the authors created the first attribute-based keyword search with efficient user revocation (ABKSUR). In their scheme, the attribute corresponds to three values, including positive, negative and "don't care". They use the authorized keyword search in which the data owner generates the file index for searching while the file is encrypted. The CSP compares the file index and the trapdoor from the user who wants to search the file with keywords, and then outputs the comparison result as validity or failure. In their scheme, it used the proxy re-encryption to achieve the user revocation, and used the lazy re-encryption (LRE) to efficiently update the file index. For LRE, the re-encryption keys are stored in CSP. The re-encryption procedures for the files will not be carried out by CSP until the user searches them. For efficiency, CSP can aggregate the newest re-encryption keys to perform once instead of many times. The scheme also has the verification part, and the data owner can use bloom filter, inverted index, hash and signature to create the authenticated data structure. Therefore, the user can verify the result from CSP by the signature of authenticated data structure.

In 2016, Ahuja et al. [1] proposed a ciphertext-policy attribute-set-based encryption with the keyword search and revocation. In their scheme, the subset concept is used to veil the information. The leaf node attributes will be obfuscated by the subset. Their scheme applied hierarchical structure that is similar to the one in HASBE. A central authority distributes the key to the domain authorities. Domain authorities verify user's signature; if it is correct, the attribute keys will be distributed to the users.

\section{Preliminaries}

\subsection{Bilinear Maps}

Definition 1 (Bilinear Maps). Let $\mathrm{G}$ and $\mathrm{G}_{\mathrm{T}}$ be multiplicative cyclic bilinear group of prime order. For $u, v \in \mathrm{G}$, and $a, b \in \mathrm{Z}_{p}$, and $g$ is the generate of $\mathrm{G}$. There are three properties: computability, non-degeneracy and bilinearity.

Computability: There exists an efficient algorithm to compute the pairing.

Non-degeneracy: $\mathrm{e}(g, g) \neq 1$.

Bilinearity : $\mathrm{e}\left(u^{a}, v^{b}\right)=\mathrm{e}(u, v)^{a b}$.

\subsection{Linear Secret-Sharing Schemes[12]}

Definition 2 (LSSS). A secret-sharing scheme $\Pi$ over a set of parties $\mathrm{P}$ is called linear (over $\mathrm{Z}_{p}$ ) if

1. The shares for each party form a vector over $Z_{p}$.

2. There exists a share-generating matrix $\mathrm{M}$ with $l$ rows and $n$ columns for $\Pi$. For all $i=1, \ldots, l$, the $i$ th row of $\mathrm{M}$ is given the label of $\rho(i)$, in which the function $\rho$ defines the party of row $i$ in M. Given a column vector $v=\left(s, r_{2}, \ldots, r_{n}\right)$, where $s \in \mathrm{Z}_{p}$ is the secret to be shared and $r_{2}, \ldots, r_{n} \in \mathrm{Z}_{p}$ are randomly generated. Then $\mathrm{M} v$ is the vector of $l$ shares of the secret s according to $\Pi$. The share $\lambda_{i}=(\mathrm{M} v)_{i}$ belongs to party $\rho(i)$.

As in [2], any LSSS satisfied as above achieves the linear reconstruction property. Suppose that $\Pi$ is an LSSS for the access structure $A$. Let $S \in A$ be an authorized set presented as $(\mathrm{M}, \rho)$, and $I \subset\{1,2,3 \ldots, m\}$ be defined as $I=\{i: \rho(i) \in S\}$. There must exist $\omega_{i} \in \mathrm{Z}_{p}$ such that $\sum_{i \in I} \omega_{i} \lambda_{i}=$ secret $s$, if $\lambda_{i}$ is a valid share in $s$ according to $\Pi$. All $\omega_{i}$ 's can be found in polynomial time in the size of the share-generating matrix M. 


\section{The Proposed Scheme}

\subsection{Scenario}

Our scheme is illustrated as Fig. 1. There are four entities: cloud service provider (CSP), trusted third party (TTP), data owner and user. The CSP can store all ciphertexts provided to the user for query. The TTP can distribute the keys to CSP and users. The data owner can upload the file to the cloud and decide the access policy. The user is a person who wants to get the file.

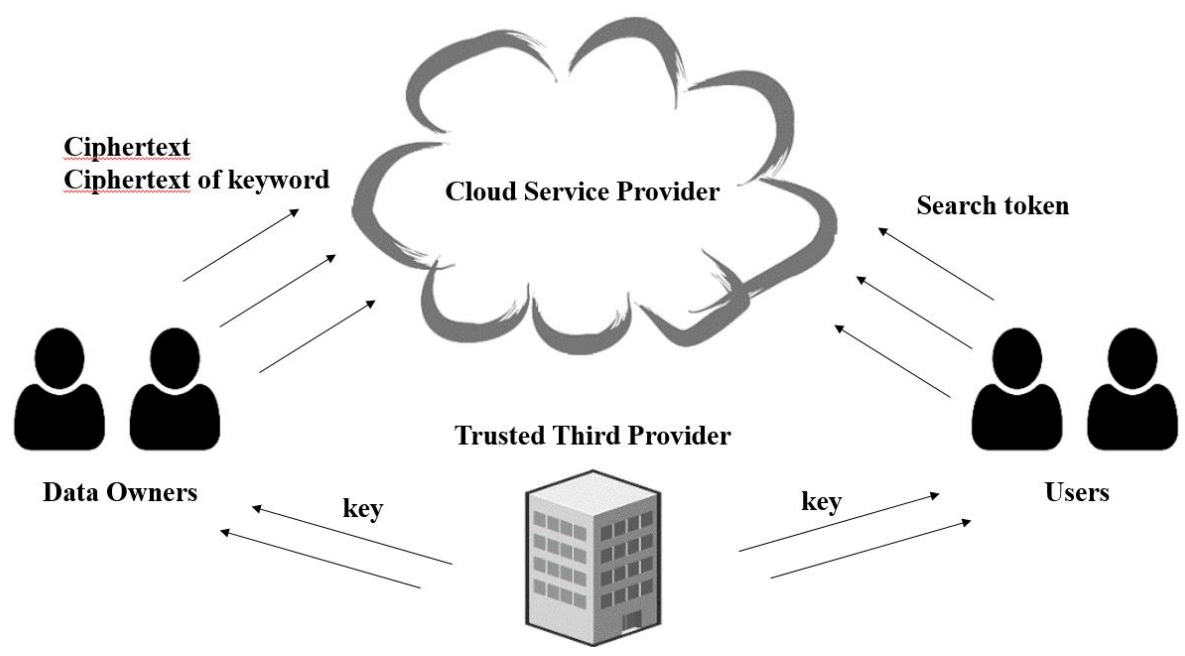

Fig. 1 Scenario of our system

In order to enhance the efficiency of access structure and achieve the attribute revocation of keyword search, our proposed scheme, based on [13] and [14], proposes a CP-ABKS with efficient revocation by using LSSS. In our scheme, CSP, delegated by the data owner, re-encrypts the access structure of the keyword search. Thus, the computation for data owner can be reduced.

\subsection{Algorithms}

Moreover, we improve the access structure format to LSSS for efficiency. There are six algorithms in our model. We discuss them as follows.

At first, TTP runs the Setup algorithm. It generates the system public key $(P K)$ and master key $(M K)$, and then publishes $P K$. Two random numbers $\alpha_{1}$ and $\alpha_{2}$ are generated for the re-encryption. $\alpha_{1}$ is associated with user secret key and $\alpha_{2}$ is associated with cloud server's secret key. They can be combined to $\alpha_{3}$, concealed into

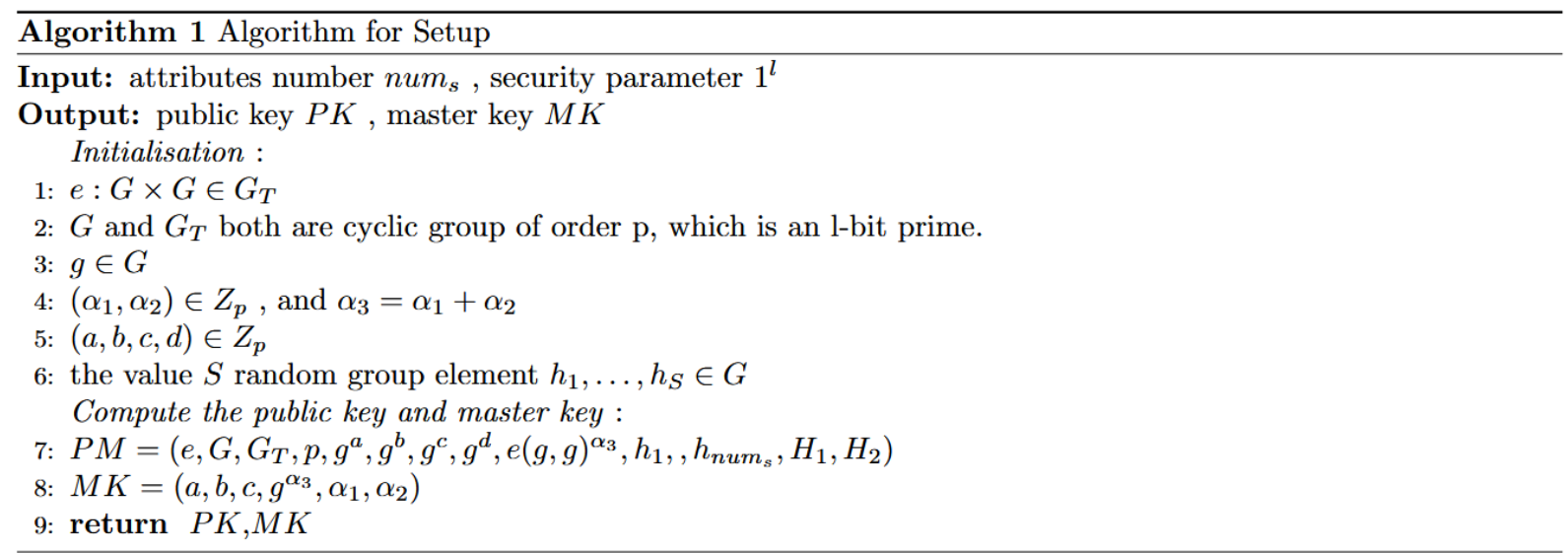

PK. 
KeyGen algorithm is ran by TTP. It outputs the secret key for user according to user's attributes. For the revocation of the system, the cloud server needs a secret key to re-encrypt the ciphertext. And the variable $A$ in the key of the user is generated for keyword search.

If data owner wants to upload a file to CSP, she/he needs to run Enc_word algorithm. This algorithm outputs a ciphertext of keyword that includes the attributes policy and some variables to be used for query. The access policy constructed by LSSS technology which can make the decryption process more efficient.

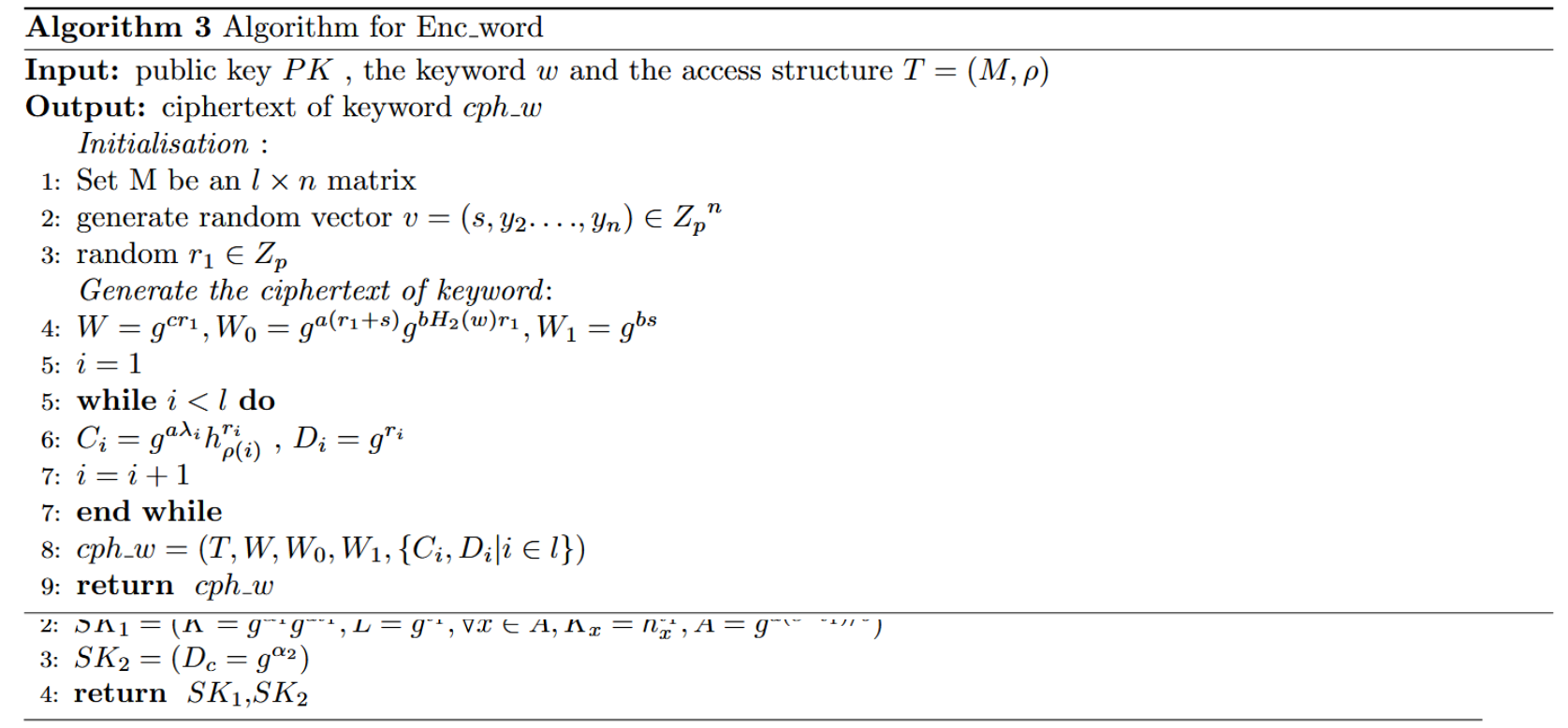

If data owner wants to change the access policy of encrypted keyword, she/he needs to send the new access structure to CSP. CSP will run the Re_enc algorithm to output a new ciphertext of password $c p h h_{-}^{\prime} w$. Without

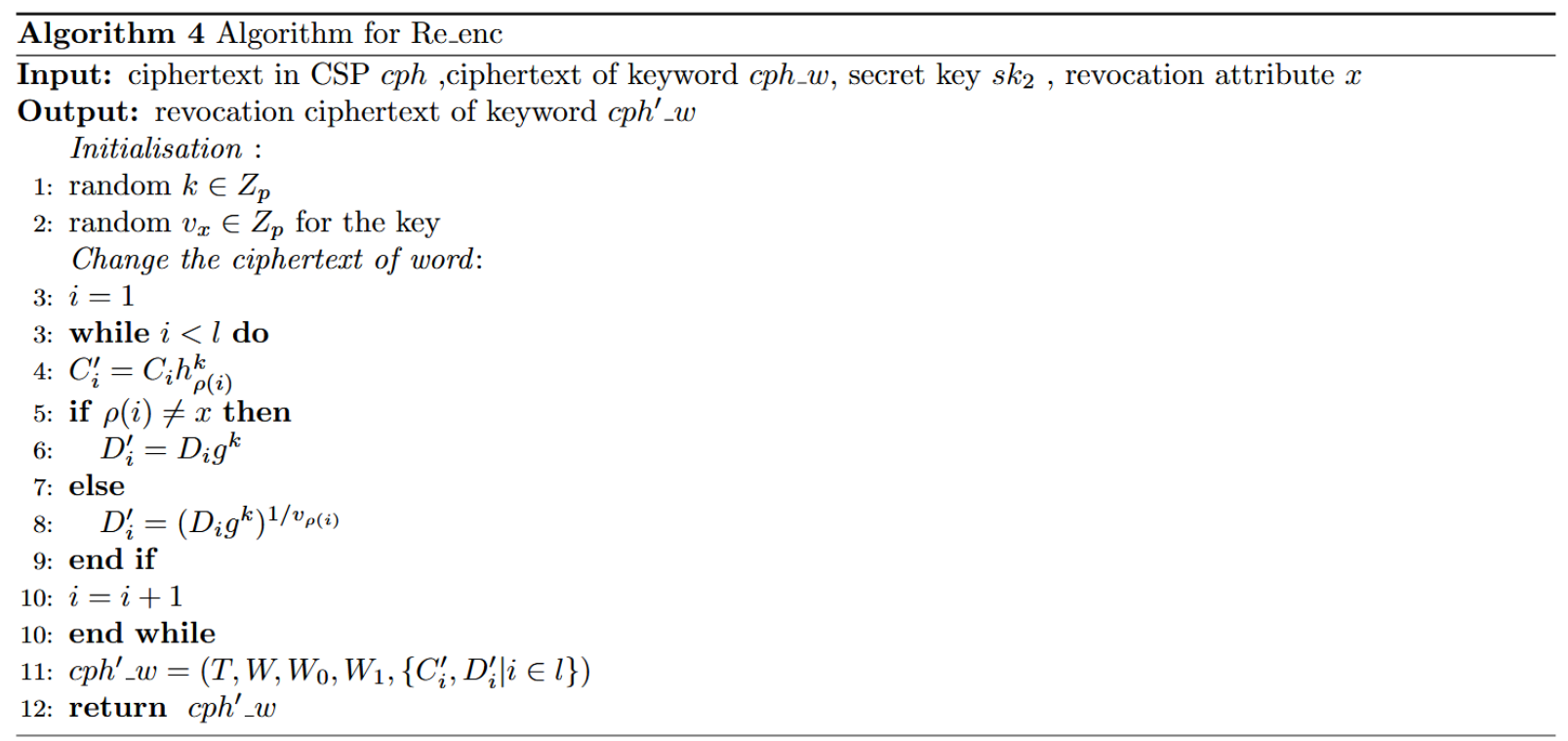

revocation procedure, the data owner needs to remove the original ciphertext and encrypt the same file according to the new access policy. In our scheme, CSP can perform the re-encrypt algorithm to change the part of the revoked attribute values. 
There may be many ciphertexts in CSP. The TokenGen algorithm is used for the user to generate the query token for a specific keyword. In this algorithm, the user generates some variables related to the keyword. These variables conceal user's keys to prove that the user has the authority to query the file. To prevent revealing of user's key to CSP on the search phase, the user's key is concealed in the exponent of the query token. The user sends the token to CSP.

On the search phase, CSP runs Search algorithm. At first, CSP compares the attributes of the token and the access structure in the ciphertext of keyword. If they are consistent, the algorithm will go on. CP then calculates the temporary key $s^{\prime}$ from user's token. And CSP will check the formula $e\left(W_{0}, t k_{2}\right)=e\left(W, t k_{1}\right) s^{\prime} e\left(t k_{3}, W_{1}\right)$. If it holds, CSP returns the desired ciphertext. Otherwise, CSP returns null.

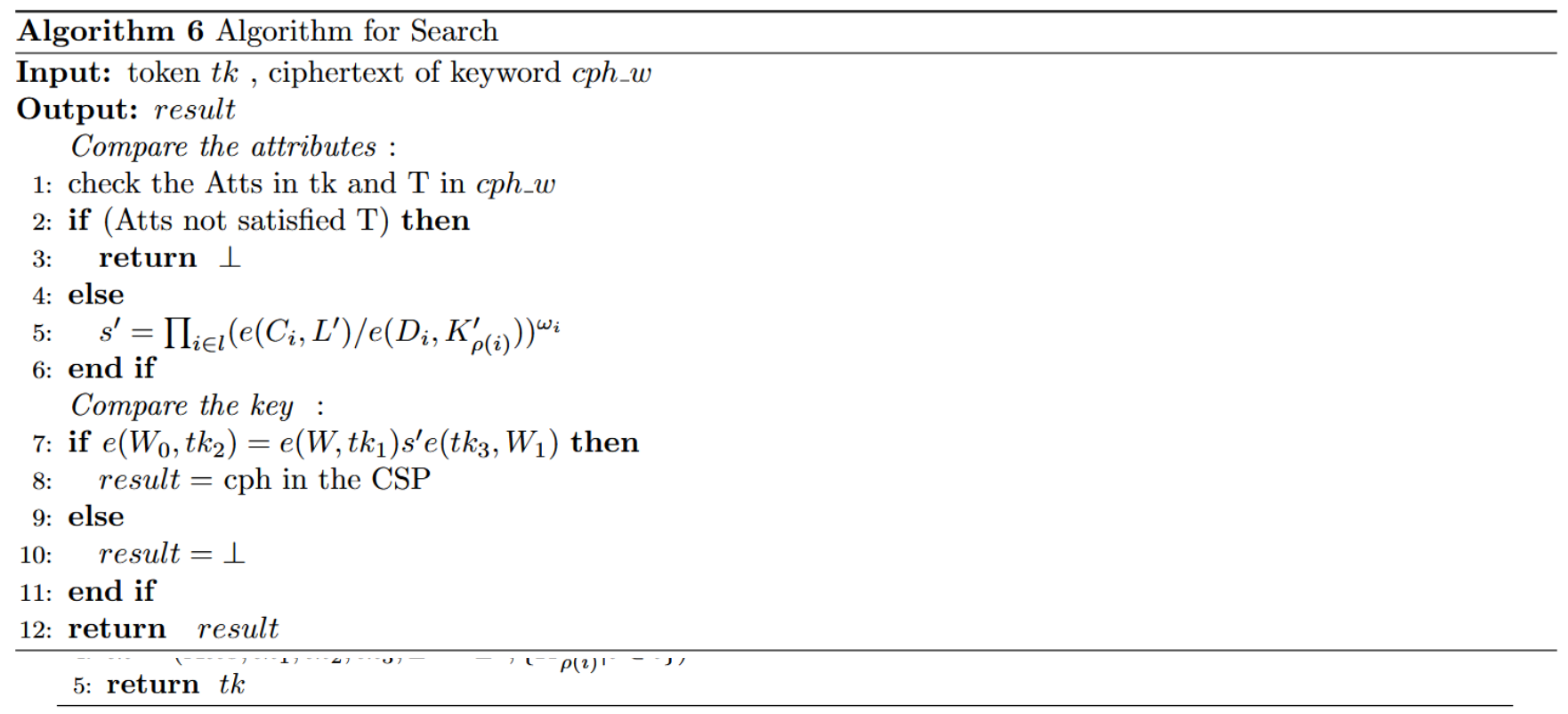

\section{Analysis}

In this section, we discuss the computation complexity and functionalities of our system. In Table 1, there are some variables of calculating computation complexity, including pairing operation: $\mathrm{P}$, exponentiation of $\mathrm{G}$ : E, exponentiation of $\mathrm{G}_{1}$ : $\mathrm{E}_{1}$, group multiplication exponentiation of $\mathrm{G}_{1}: \mathrm{M}_{1}$, number of all attributes: num , number of user's attributes: $U_{s}$, number of ciphertext's attributes: $T_{s}$, hash function operation: $H_{1}$ and hash function operation: $\mathrm{H}_{2}$. Compared with [13] and [10], our scheme has an advantage over [13] in Search part and we have an advantage over [10] in Setup part.

TABLE I: Computation comparison

\begin{tabular}{|l|l|l|l|}
\hline & Our Scheme & {$[13]$} & {$[10]$} \\
\hline Setup & $\mathrm{P}+6 \mathrm{E}+\mathrm{E}_{1}$ & $3 \mathrm{E}$ & 3 num $_{s} \mathrm{E}+\mathrm{E}_{1}+\mathrm{P}$ \\
\hline KeyGen & $\left(4+\mathrm{U}_{\mathrm{s}}\right) \mathrm{E}$ & $\left(2 \mathrm{U}_{\mathrm{s}}+2\right) \mathrm{E}+\mathrm{U}_{\mathrm{s}} \mathrm{H}_{1}$ & $\left(2 \mathrm{num}_{\mathrm{s}}+1\right) \mathrm{E}+2 \mathrm{E}_{1}$ \\
\hline Enc_word & $\mathrm{H}_{2}+\left(4+3 \mathrm{~T}_{\mathrm{s}}\right) \mathrm{E}$ & $\left(2 \mathrm{~T}_{\mathrm{s}}+4\right) \mathrm{E}+\mathrm{T}_{\mathrm{s}} \mathrm{H}_{1}$ & $\left(\right.$ num $\left._{\mathrm{s}}+1\right) \mathrm{E}+\mathrm{E}_{1}$ \\
\hline Re_word & $2 \mathrm{~T}_{\mathrm{s}} \mathrm{E}$ & No & $\mathrm{T}_{\mathrm{s}} \mathrm{E}$ \\
\hline TokenGen & $\mathrm{H}_{2}+\left(3+\mathrm{U}_{\mathrm{s}}\right) \mathrm{E}$ & $\left(2 \mathrm{U}_{\mathrm{s}}+4\right) \mathrm{E}$ & $\left(2 \mathrm{num}_{\mathrm{s}}+1\right) \mathrm{E}$ \\
\hline Search & $\left(2 \mathrm{~T}_{\mathrm{s}}+3\right) \mathrm{P}+\mathrm{E}_{1}$ & $\left(2 \mathrm{~T}_{\mathrm{s}}+3\right) \mathrm{P}+\mathrm{T}_{\mathrm{s}} \mathrm{E}_{1}$ & $\left(\right.$ num $\left._{\mathrm{s}}+1\right) \mathrm{P}+\left(\right.$ num $\left._{\mathrm{s}}+2\right) \mathrm{M}_{1}+\mathrm{E}_{1}$ \\
\hline
\end{tabular}

In table 2, we discuss the functionality of different schemes. Our scheme is a CP-ABE under LSSS structure with keyword search and revocation. Compared with [13], our scheme has the superiorities of LSSS structure and revocation. The paper [14] has the revocation and LSSS structure, but cannot perform keyword search. In [10], although it has keyword search and revocation, the efficiency is lower due to the tree-type access structure. 
TABLE II: Functionality comparison

\begin{tabular}{|l|l|l|l|l|}
\hline & Our Scheme & {$[13]$} & {$[14]$} & {$[10]$} \\
\hline ABE & CPABE & CPABE & CPABE & CPABE \\
\hline Structure & LSSS & Tree & LSSS & Tree \\
\hline Keyword Search & Yes & Yes & No & Yes \\
\hline Revocation & Yes & No & Yes & Yes \\
\hline
\end{tabular}

\section{Conclusion}

In this paper, we have proposed a CP-ABKS with efficient revocation. In our scheme, CSP can run the Re_word algorithm to re-encrypt the access structure of the keyword. Our proposed scheme is superior to the previous works. In [13], keyword search encryption can be efficiently performed, but their scheme cannot support revocation procedure on the keywords. If the data owner want to change the access structure on the ciphertext and keyword, she/he must be encrypt the file and access structure again. In [10], CSP can carry out both ciphertext revocation and keyword search revocation, but they used tree-type with polynomial-based access structure instead of LSSS to make it less efficient on decryption.

\section{Acknowledgements}

This research is supported by Ministry of Science and Technology, Taiwan under the grant: MOST 1052221-E-415-015.

\section{References}

[1] R. Ahuja, S. K. Mohanty, and K. Sakurai, "An identity preserving access control scheme with flexible system privilege revocation in cloud computing," The 12th Asia Joint Conference on Information Security (AsiaJCIS), 2016. https://doi.org/10.1109/AsiaJCIS.2016.23

[2] A. Beimel, "Secure schemes for secret sharing and key distribution," Ph.D. dissertation, Israel Institute of Technology, Technion, Haifa, Israel, 1996.

[3] J. Bethencourt, A. Sahai, and B. Waters, "Ciphertext-policy attribute-based encryption," IEEE Symposium on Security and Privacy, 2007. SP'07, pp. 321-334, 2007.

https://doi.org/10.1109/SP.2007.11

[4] D. Boneh, C. Di, R. Ostrovsky, and G. Persiano, "Public key encryption with keyword search," Advances in Cryptology - EUROCRYPT 2004, Lecture Notes in Computer Science, vol. 3027, pp. 506-522, 2004. https://doi.org/10.1007/978-3-540-24676-3_30

[5] Q. Dong, Z. Guan, and Z. Chen, "Attribute-based keyword search efficiency enhancement via an online/offline approach," 21st International Conference on Parallel and Distributed Systems (ICPADS), IEEE Press, pp. 298-305, 2015.

[6] M. Li, S. Yu, N. Cao, and W. Lou, "Authorized private keyword search over encrypted data in cloud computing," 31st International Conference on Distributed Computing Systems (ICDCS),. IEEE Press, pp. 383-392, 2011. https://doi.org/10.1109/ICDCS.2011.55

[7] K. Liang, L. Fang, W. Susilo, and D. S. Wong, "A ciphertext-policy attribute-based proxy re-encryption with chosenciphertext security," 5th International Conference on Intelligent Networking and Collaborative Systems (INCoS), IEEE Press, 2013. https://doi.org/10.1109/INCoS.2013.103

[8] X. Liang, X. Li, R. Lu, X. Lin, and X. Shen, "An efficient and secure user revocation scheme in mobile social networks,” 2011 Global Telecommunications Conference (GLOBECOM 2011), IEEE Press, pp. 1-5, 2011.

https://doi.org/10.1109/GLOCOM.2011.6134273 
[9] A. Sahai, and B. Waters, "Fuzzy identity-based encryption," Advances in Cryptology - EUROCRYPT 2005, Lecture Notes in Computer Science, vol. 3494, pp. 457-473, 2005.

https://doi.org/10.1007/11426639_27

[10] W. Sun, S. Yu, W. Lou, Y. T. Hou, and H.Li, "Protecting your right: verifiable attribute-based keyword search with fine-grained owner-enforced search authorization in the cloud," IEEE Transactions on Parallel and Distributed Systems 27.4 (2016): 1187-1198.

https://doi.org/10.1109/TPDS.2014.2355202

[11] Z. Wan, J. E. Liu, and R. H. Deng, "HASBE: a hierarchical attribute-based solution for flexible and scalable access control in cloud computing,” IEEE Transactions on Information Forensics and Security 7.2 (2012): 743-754. https://doi.org/10.1109/TIFS.2011.2172209

[12] B. Waters "Ciphertext-policy attribute-based encryption: An expressive, efficient, and provably secure realization," Public Key Cryptography, vol. 6571, 2011.

[13] Q. Zheng, S. Xu, and G. Ateniese, "VABKS: verifiable attribute-based keyword search over outsourced encrypted data," in Proc. INFOCOM 2014, IEEE Press, 2014.

https://doi.org/10.1109/INFOCOM.2014.6847976

[14] L. Zu, Z. Liu, and J. Li, "New ciphertext-policy attribute-based encryption with efficient revocation," 2014 17th International Conference on Computer and Information Technology (ICCIT), IEEE Press, 2014.

https://doi.org/10.1109/CIT.2014.97 